\title{
Proton transfer and kinetic isotope effect in Morita-Baylis-Hillman reaction under solvent effects. A detailed computational study
}

Veejendra K. Yadav*

Department of Chemistry, Indian Institute of Technology Kanpur, Kanpur 208016, India

vijendra@iitk.ac.in

\begin{abstract}
Solvent effects of $\mathrm{CH}_{2} \mathrm{Cl}_{2}, \mathrm{CH}_{3} \mathrm{CN}$, THF and DMSO on the profile of the Morita-Baylis-Hillman $(\mathrm{MBH})$ reaction were discovered to lower the activation energy of aldol step. Proton-transfer through seven-membered TS state structure enroute Hofmann elimination and also the four-membered TS structure are sufficiently low energy processes in comparison to aldol reaction and, hence, unlikely to contribute to the overall kinetics of the reaction. As an exception, proton-transfer through sevenmembered TS structure constitutes the rate-controlling event for the DABCO-catalyzed reaction of methyl acrylate with $p$-nitrobenzaldehyde under the solvent effects of DMSO. The acetal route was not found to contribute to kinetic isotope effect, which has otherwise been measured to be as high as $5.2 \pm 0.6$. The simultaneous proton-transfer to the aldol-derived alkoxide and abstraction of proton from the $\alpha$-carbon of activated alkene by hydroxylic solvents was found to be barrier-less. The reported catalysis by hydroxylic solvents is therefore likely by lowering the activation energy of the aldol step due to activation of aldehyde possibly by protonation or hydrogen-bonding. The present computational results are in excellent agreement with the more than three decade old experimental findings of Hill and Isaacs. These authors have reported the aldol step as rate-limiting and absolutely no kinetic isotope effect.
\end{abstract}

Key words: Morita-Baylis-Hillman reaction, solvent effects, catalysis by hydroxylic solvents, proton transfer, kinetic isotope effect

Introduction. The MBH reaction is generally believed to follow the pathway given in Scheme 1 by using $\mathrm{Me}_{3} \mathrm{~N}(\mathbf{1})$ as the organocatalyst, cyclopentenone (2) as the activated alkene, and acetaldehyde (4) as the aldol partner. ${ }^{1}$ Reversible conjugate addition of $\mathrm{Me}_{3} \mathrm{~N}$ to cyclopentenone generates the enolate 3 , which enters aldol reaction with the aldehyde $\mathbf{4}$ and forms the zwitterion $\mathbf{5}$. Now, proton transfer from the $\alpha$ position to alkoxide through four-membered transition state (TS) structure generates yet another zwitterion 6a, which undergoes $\mathrm{E} 1 \mathrm{cB}$ reaction to form the product $\mathbf{7}$ and liberate $\mathrm{Me}_{3} \mathrm{~N}$ to begin with the reaction all over again. ${ }^{2}$ 


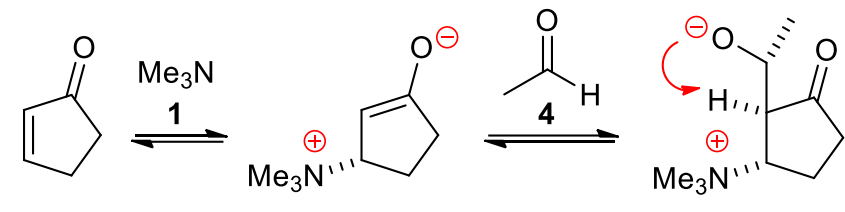

2

3

5

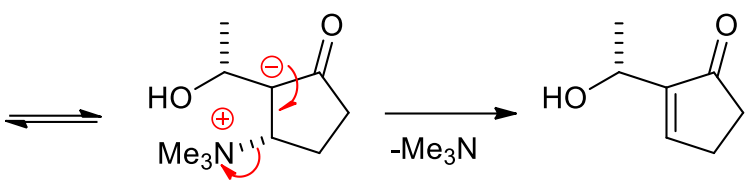

$6 a$

7

Scheme 1. The generally accepted pathway for the $\mathrm{Me}_{3} \mathrm{~N}$-catalysed $\mathrm{MBH}$ reaction of cyclopentenone with acetaldehyde

Hill and Isaacs studied the kinetics of the reaction of $\alpha^{-2} \mathrm{H}$ acrylonitrile with acetaldehyde under catalysis by DABCO and suggested the aldol step to be rate-limiting. ${ }^{3}$ However, on the basis of reaction rate data for the DABCO-catalysed reaction of methyl acrylate with aryl aldehydes, McQuade has proposed a new mechanism for the Baylis-Hillman reaction involving the formation of a hemiacetal intermediate and determined that the rate determining step was second order in the aldehyde and first order in DABCO and acrylate. ${ }^{4}$ McQuade observed further that this mechanism was general to aryl aldehydes under polar, nonpolar, and protic conditions using both rate data and isotope effect experiments. Aggarwal also studied the quinuclidine-catalysed $\mathrm{MBH}$ reaction of ethyl acrylate with benzaldehyde, evaluated the reaction mechanism by kinetic and theoretical means, and proposed the proton transfer step $\mathbf{5} \rightarrow \mathbf{6 a}$ as rate-limiting because it was sufficiently energy demanding due to the strain induced in attaining the eclipsed four-membered ring transition state (TS) structure. ${ }^{2 b} \mathrm{~A}$ significant finding of this study was also the observation that all equilibria prior to thermodynamically favorable liberation of the MBH product lay predominantly on the side of the starting materials. In 2015, Singleton and Plata showed that the reaction conditions determined the limiting step of the reaction. ${ }^{2 e}$ Proton-transfer was suggested as the primary rate-limiting step at $25{ }^{\circ} \mathrm{C}$, but the aldol step was partially rate-limiting and became the primary ratelimiting step at low temperatures.

Intermolecular proton-transfer, wherein an aldol-derived alkoxide abstracts $\alpha-\mathrm{H}$ from another such species or two alkoxide species mutually exchange protons through an eight-membered ring TS structure, is also a possibility, but not explored till date!

We have recently proposed an alternate significantly lower energy pathway shown in Scheme 2 from computations in gas phase at M06-2X/6-311++G(d,p) level of theory. ${ }^{5 a}$ We have also shown that DABCO, which would lead to a bridge-head ylide in following this pathway, also participated in the reaction with 
just about the same ease as $\mathrm{Me}_{3} \mathrm{~N}^{5 b}$ The enolate 3, formed from conjugate addition of $\mathrm{Me}_{3} \mathrm{~N}$ to cyclopentenone via the TS structure TS1, combines with $\mathrm{CH}_{3} \mathrm{CHO}$ in aldol fashion via the TS structure resembling TS2 to generate the zwitterion $\mathbf{5}$. The alkoxide function in $\mathbf{5}$ pulls a proton from one of the methyl groups on nitrogen through a seven-membered TS structure resembling TS3 and forms the ylide 6b. Now, Hofmann elimination ${ }^{6}$ through the TS structure TS4 delivers the product 7. The anti-arrangement of the enolate and carbonyl groups in TS2 avoids steric interactions. ${ }^{7}$

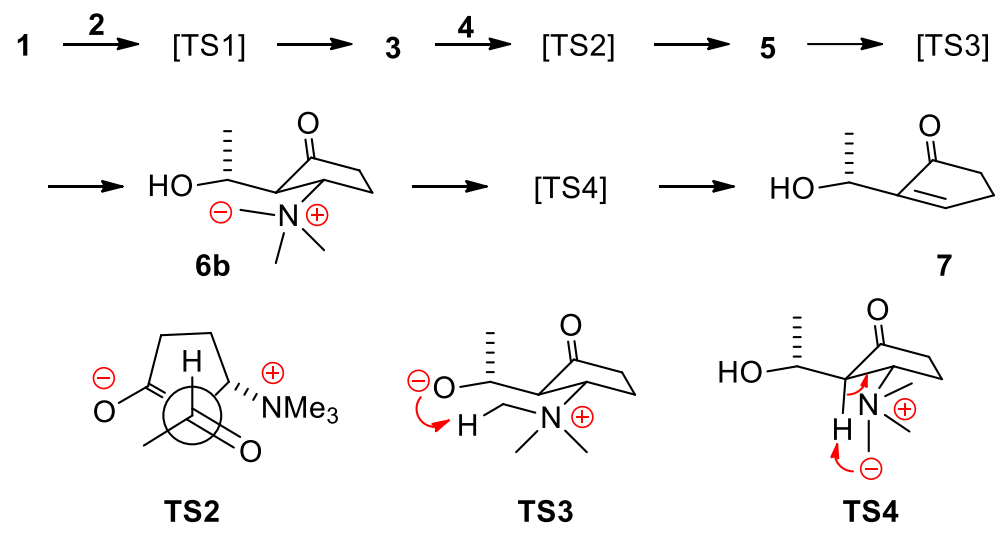

Scheme 2. The Hofmann elimination pathway for the $\mathrm{Me}_{3} \mathrm{~N}$-catalysed $\mathrm{MBH}$ reaction of cyclopentenone and acetaldehyde

In the above investigation, the choice of cyclopentenone as activated alkene was guided by limiting the conformational flexibility of the enolate formed from conjugate addition of the amine. Since it has been amply demonstrated in the literature that the rate limiting step is either the carbon-carbon bond formation during the aldol step or proton transfer in the subsequent step or a combination of both depending on the reaction conditions, we focussed on the proton transfer step $5 \rightarrow 6 a$ in Scheme 1 and $\mathbf{6 b} \rightarrow$ TS4 $\rightarrow \mathbf{7}$ in Scheme 2, and estimated their relative ease of occurrence in comparison to the aldol reaction $(\mathbf{3}+\mathbf{4}) \rightarrow$ TS2 $\rightarrow \mathbf{5}$ from their activation energies. The profile of the conjugate addition step (1+2) $\rightarrow$ TS1 $\rightarrow \mathbf{3}$ was also estimated to generate the entire reaction profile shown in Figure 1. 


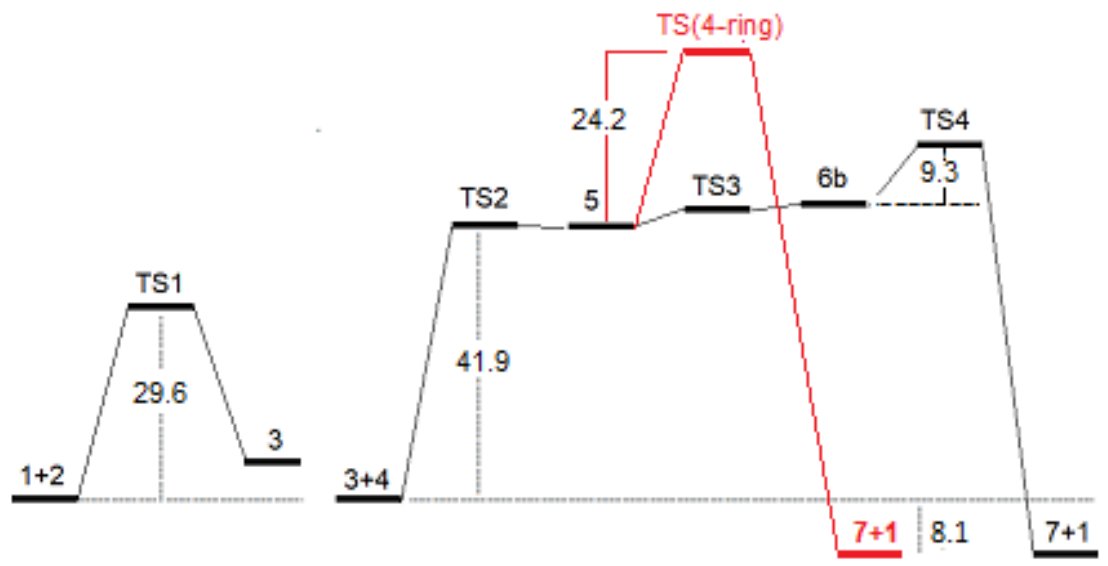

Figure 1. The relative profile of the $\mathrm{M}_{3} \mathrm{~N}$-catalyzed $\mathrm{MBH}$ reaction of cyclopentenone with acetaldehyde in gas phase. TS (4-ring) is the TS for the conversion $\mathbf{5} \rightarrow \mathbf{6 a}$. The activation free energies are in $\mathrm{kcal} / \mathrm{mol}$.

The activation free energy $\left(\Delta G^{\ddagger}\right)$ of the aldol step $(3+4) \rightarrow$ TS2 $\rightarrow \mathbf{5}$ was estimated to be the largest at $41.9 \mathrm{kcal} / \mathrm{mol}$. The initial conjugate addition $(\mathbf{1}+\mathbf{2}) \rightarrow$ TS1 $\rightarrow$ 3, proton transfer $\mathbf{5} \rightarrow$ TS3 $\rightarrow$ 6b, and Hofmann elimination $\mathbf{6 b} \rightarrow$ TS4 $\rightarrow 7$ required relatively much lower energies such as 29.6, 2.6 and 9.3 $\mathrm{kcal} / \mathrm{mol}$, respectively. Except the last step, all other steps were mildly to sufficiently endergonic and, hence, reversible as observed by Aggarwal previously. While the last step was exergonic by a whopping $52.9 \mathrm{kcal} / \mathrm{mol}$, the overall process $(1+2+4) \rightarrow(1+7)$ was exergonic by only $2.5 \mathrm{kcal} / \mathrm{mol}$, which may be responsible for the overall sluggish nature of the reaction, and also the reaction forward. An increase in reaction temperature is, therefore, likely to switch on the equilibrium to the reactants to make the reaction less efficient as indeed observed. ${ }^{8}$

In comparison, $\Delta G^{\ddagger}$ for the proton-transfer step $\mathbf{5} \rightarrow \mathbf{6 a}$ through four-membered TS structure was estimated at $24.2 \mathrm{kcal} / \mathrm{mol}$ against $9.3 \mathrm{kcal} / \mathrm{mol}$ for the Hofmann elimination $\mathbf{6 b} \rightarrow$ TS4 $\rightarrow 7$. Since the proton transfer $\mathbf{5} \rightarrow \mathrm{TS} 3 \rightarrow \mathbf{6 b}$ was extremely rapid for very low $\Delta G^{\ddagger}$, the pathway shown in Scheme 2 was claimed to offer an alternate lower energy pathway than the previously popular pathway outlined in Scheme 1.

Another important lesson learnt from the above exercise was about the kinetic isotope effect $k_{H} / k_{D}$ for proton transfer from $\alpha$-position of the activated alkene. The aldol reaction $(3+\mathbf{4}) \rightarrow$ TS2 $\rightarrow \mathbf{5}$ being the slowest, the reaction is not expected to show any kinetic isotope effect (vide infra). Still, among the reactions following aldol, the product-yielding Hofmann elimination $\mathbf{6 b} \rightarrow$ TS4 $\rightarrow \mathbf{7}$, which involves $\alpha$ proton transfer, is the slowest and, thus, it may display kinetic isotope effect locally. The $k_{H} / k_{D}$ was estimated at 1.26 from the negative frequencies of the respective TS structures. ${ }^{9}$ 
$\mathrm{MBH}$ reaction in the absence of solvents has been reported to be significantly fast in comparison to those in solvents. Mack and Shumba obtained $>98 \%$ yield of the product from methyl acrylate and $p$ nitrobenzaldehyde under DABCO-catalysis in as little as $30 \mathrm{~min}$ by solvent-free mechanochemistry. ${ }^{10}$ Saikia and Sarma have reported remarkable rate acceleration under solvent-free conditions and obtained high yields of the products in short reaction times. ${ }^{11}$ Moghadam and Miri have obtained the adducts of isatin derivatives in high yields under DABCO-catalysis and solvent-free conditions at $0{ }^{\circ} \mathrm{C} .{ }^{12}$ Zhao et. al. have reported $86 \%$ yield of a $\mathrm{MBH}$ product under solvent-free conditions as use of solvents such as acetonitrile and tetrahydrofuran resulted in only $30-39 \%$ yields. ${ }^{13}$ Thus, it was hypothesized that solvents were mainly used to solubilize nonhomogeneous reaction mixtures and promote zwitterionic species. For this reason, polar and protic solvents were considered appropriate for the reaction. ${ }^{14}$

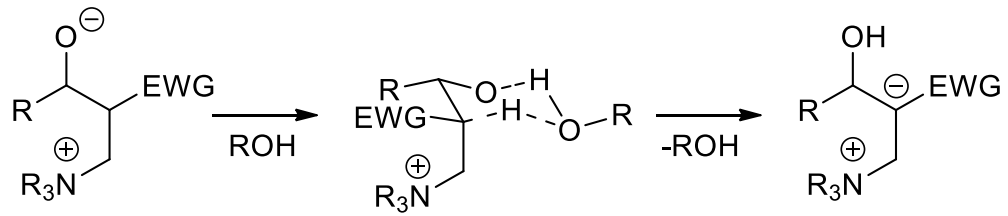

Scheme 3. Alcohol- and water-mediated simultaneous proton-transfer and proton-abstraction proposed by Aggarwal

Since protic solvents such as water and alcohol were recognized to catalyse the reaction by simultaneously mediating proton-transfer to the alkoxide $\mathbf{5}$ and also proton-abstraction from the $\alpha$-position of activated alkene through a six-membered TS structure as shown in Scheme $3,{ }^{2 \mathrm{~d}}$ we became interested in studying (a) the effects of aprotic solvents such as methylene chloride $\left(\mathrm{CH}_{2} \mathrm{Cl}_{2}\right)$, acetonitrile $\left(\mathrm{CH}_{3} \mathrm{CN}\right)$, tetrahydrofuran (THF) and dimethyl sulfoxide (DMSO) on the overall reaction profile, and the associated kinetic isotope effects and (b) catalysis by hydroxylic solvents such as $\mathrm{H}_{2} \mathrm{O}$ and $\mathrm{MeOH}$, and the associated kinetic isotope effects.

The study of kinetic isotope effect $k_{H} / k_{D}$ was warranted by the observation of as high a value as $5 \pm 2$ from competition experiments between methyl acrylate and methyl $\alpha^{2} \mathrm{H}$ acrylate in the absence of solvent at the initial stage of the reaction ( $<20 \%$ conversion) by Aggarwal ${ }^{2 \mathrm{~d}}$ and a similar value $5.2 \pm 0.6$ by McQuade ${ }^{4}$ from DABCO-catalyzed reaction of methyl $\alpha-{ }^{2} \mathrm{H}$ acrylate and $p$-nitrobenzaldehyde in DMSO as the reaction solvent. From our gas phase study of the reaction above, such a high value was never estimated and, hence, it required a detailed exploration. 


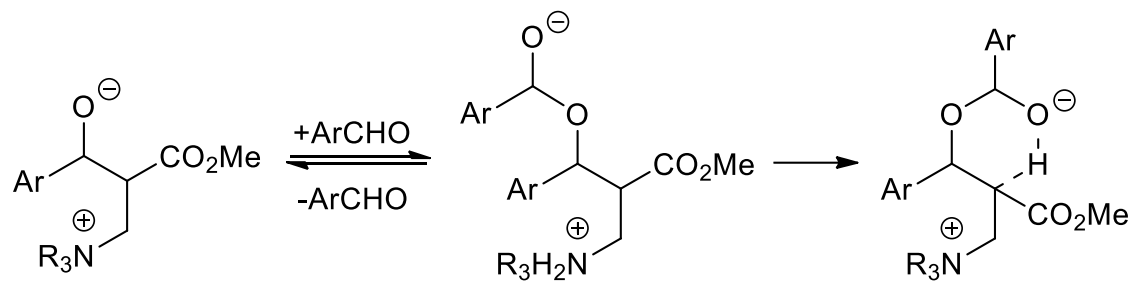

RLS= Rate Limiting Step

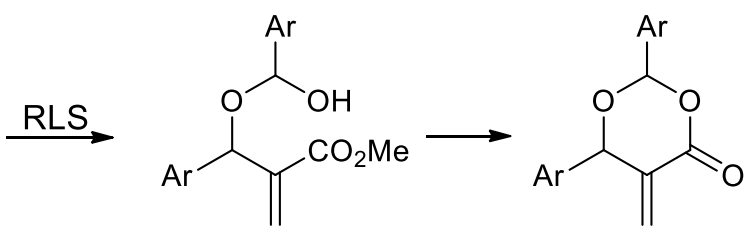

Scheme 4. McQuade's observation of $3^{\text {rd }}$ order kinetics of the MBH reaction [RLS = Rate Limiting Step]

The above study by McQuade also showed that the kinetics at the early stage of the reaction in the absence of protic species was second order in aldehyde, indicating that the rate-limiting proton-transfer step involved a second molecule of the aldehyde. ${ }^{4}$ In other words, the hemiacetal intermediate underwent proton-transfer via a six-membered TS structure as shown in Scheme 4. This proposal finds support from the isolation of a dioxane product in some instances. ${ }^{15}$ The kinetic isotope effect $k_{H} / k_{D}$ emerging from this route has also been estimated in the present study.

Computational methods: All the geometry optimizations and TS structure searches were carried out using the global hybrid meta-GGA M06-2X density functional and 6-311++G(d,p) basis set at $298.15 \mathrm{~K}$ and $1 \mathrm{~atm}$ pressure. ${ }^{16}$ The optimized structures were verified as minima or first order saddle points by harmonic vibrational frequency analysis. The solvent effects of $\mathrm{CH}_{2} \mathrm{Cl}_{2}, \mathrm{CH}_{3} \mathrm{CN}$, THF and DMSO on the reaction profiles were estimated using the Conductor Polarized Continuum Model (CPCM). ${ }^{17}$ All the energies reported herein are Gibbs free energies, i.e., sum of electronic and thermal free energies expressed in $\mathrm{kcal} / \mathrm{mol}$. The calculations were carried out using Gaussian 16 suite of programs. ${ }^{18}$

Results and Discussion. The profiles of the $\mathrm{Me}_{3} \mathrm{~N}$-catalyzed $\mathrm{MBH}$ reactions of cyclopentenone with acetaldehyde under the solvent effects of $\mathrm{CH}_{2} \mathrm{Cl}_{2}, \mathrm{CH}_{3} \mathrm{CN}$, THF and DMSO are shown in Figures 2-5, respectively. A comparison of the activation energies in these Figures with those in the gas phase given in Figure 1 highlights the followings:

(a) Solvents substantially bring down the activation energies of conjugate addition and aldol reaction. The TS structures for the these reactions are, therefore, more charged than the reactants. ${ }^{19}$ 
(b) While the reduction in $\Delta G^{\ddagger}$ for conjugate addition is in the range of 9-11 kcal/mol from that in the gas phase reaction, that of the aldol reaction is in the range $11-15 \mathrm{kcal} / \mathrm{mol}$.

(c) $\mathrm{CH}_{3} \mathrm{CN}$ and DMSO are more effective than $\mathrm{CH}_{2} \mathrm{Cl}_{2}$ and THF in bringing down $\Delta G^{\ddagger}$ for conjugate addition and aldol reaction. In fact, $\mathrm{CH}_{3} \mathrm{CN}$ and DMSO, and $\mathrm{CH}_{2} \mathrm{Cl}_{2}$ and THF are much alike in respect of the reduction in $\Delta G^{\ddagger}$.

(d) The $\Delta G^{\ddagger}$ for the proton-transfer step $\mathbf{5} \rightarrow \mathrm{TS} 3 \rightarrow \mathbf{6 b}$ is the smallest, $2.6 \mathrm{kcal} / \mathrm{mol}$, in the gas phase. This energy is substantially raised to $11.5-13.0 \mathrm{kcal} / \mathrm{mol}$ on inclusion of solvent effects. The similar contributions of $\mathrm{CH}_{3} \mathrm{CN}$ and DMSO, and $\mathrm{CH}_{2} \mathrm{Cl}_{2}$ and THF hold here as well. $\mathrm{CH}_{3} \mathrm{CN}$ and DMSO raise $\Delta G^{\ddagger}$ a little more than $\mathrm{CH}_{2} \mathrm{Cl}_{2}$ and THF. This result indicates that the TS structure for protontransfer is less charged than the reacting substrate. ${ }^{19}$

(e) The $\Delta G^{\ddagger}$ for Hofmann elimination $\mathbf{6 b} \rightarrow$ TS4 $\rightarrow \mathbf{7}$ is also the smallest, $9.3 \mathrm{kcal} / \mathrm{mol}$, in the gas phase reaction and raised by $3.0-4.0 \mathrm{kcal} / \mathrm{mol}$ under solvent effects. The TS structure is, therefore, less charged than the reactant ylide.

(f) The $\Delta G^{\ddagger}$ for proton transfer $\mathbf{5} \rightarrow \mathbf{6 a}$ via four-membered TS structure is raised merely by 1.4-2.2 $\mathrm{kcal} / \mathrm{mol}$. However, it still remains larger than those for $5 \rightarrow$ TS3 $\rightarrow$ 6b and $6 \mathrm{~b} \rightarrow$ TS4 $\rightarrow 7$ transformations.

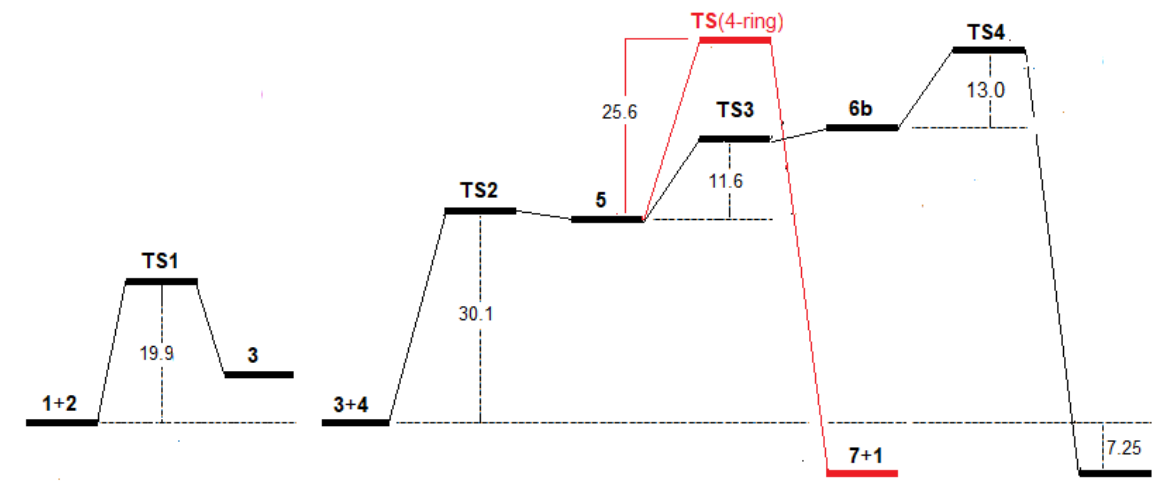

Figure 2. The relative profile of the $\mathrm{M}_{3} \mathrm{~N}$-catalysed $\mathrm{MBH}$ reaction of cyclopentenone with acetaldehyde under the solvent effects of $\mathrm{CH}_{2} \mathrm{Cl}_{2}$. TS (4-ring) is the four-membered TS structure for the transformation $\mathbf{5} \rightarrow \mathbf{6 a}$. 


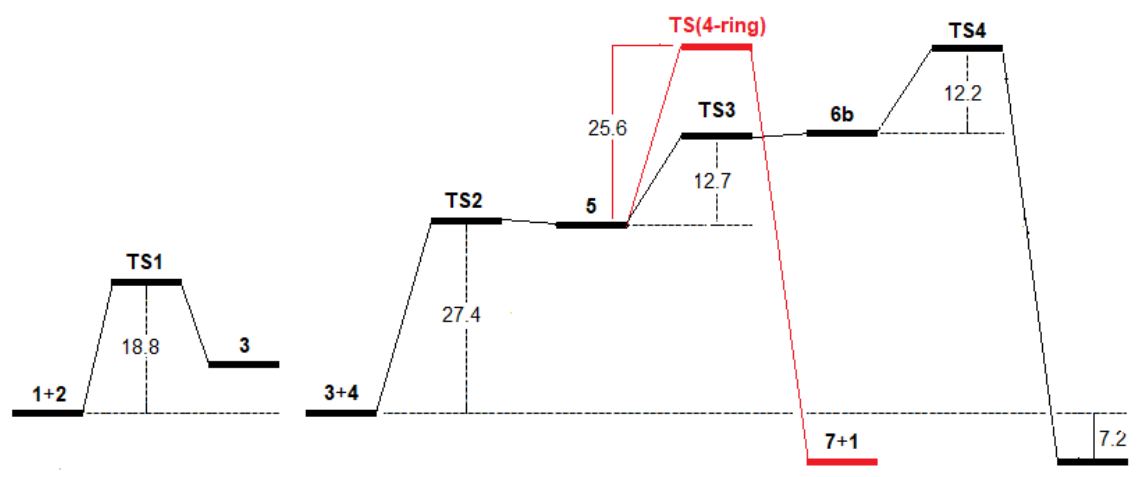

Figure 3. The relative profile of the $\mathrm{M}_{3} \mathrm{~N}$-catalysed $\mathrm{MBH}$ reaction of cyclopentenone with acetaldehyde under the solvent effects of $\mathrm{CH}_{3} \mathrm{CN}$. TS (4-ring) is the four-membered TS structure for the transformation $5 \rightarrow 6 a$.

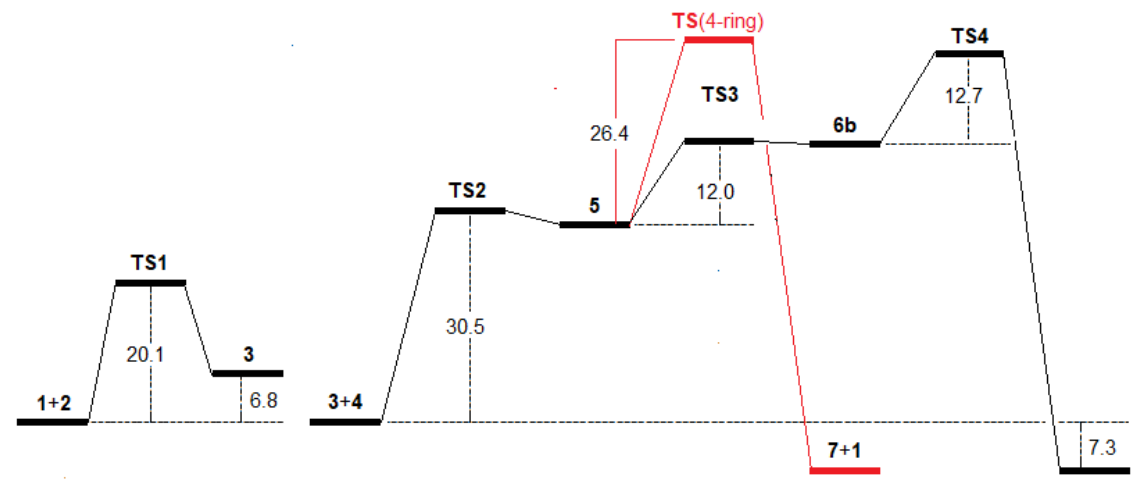

Figure 4. The relative profile of the $\mathrm{M}_{3} \mathrm{~N}$-catalysed $\mathrm{MBH}$ reaction of cyclopentenone with acetaldehyde under the solvent effects of THF. TS (4-ring) is the four-membered TS structure for the transformation $\mathbf{5} \rightarrow \mathbf{6 a}$.

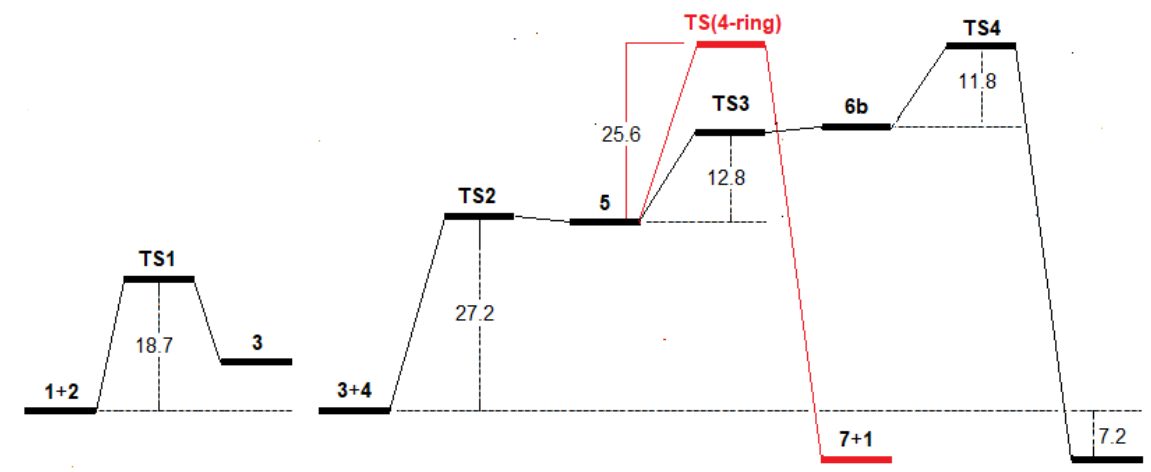

Figure 5. The relative profile of the $\mathrm{M}_{3} \mathrm{~N}$-catalysed $\mathrm{MBH}$ reaction of cyclopentenone with acetaldehyde under the solvent effects of DMSO. TS (4-ring) is the four-membered TS structure for the transformation $\mathbf{5} \rightarrow \mathbf{6 a}$.

Overall, the pathway shown in Scheme 2 is a lower energy pathway for the MBH reaction than the widely accepted mechanistic sequence shown in Scheme 1 under solvent conditions also. The aldol reaction is rate-limiting throughout as it is the highest energy requiring transformation in the entire sequence. In no solvent did the proton transfer either $5 \rightarrow \mathbf{6 a}$ or $\mathbf{5} \rightarrow \mathbf{6 b}$ ever become rate-limiting. We have collected the activation energies of various steps in Table 1 to allow a convenient comparison of the profiles. 
Table $1 . \Delta \mathbf{G}^{\ddagger}$ of various steps in Scheme 2 and also the transformation $\mathbf{5} \rightarrow \mathbf{6 a}(\mathrm{kcal} / \mathrm{mol})$ in the gas phase and also under different solvent effects

\begin{tabular}{llllll}
\hline Phase & $\Delta G_{\mathrm{TS} 1}^{\ddagger}$ & $\Delta G_{\mathrm{TS} 2}^{\ddagger}$ & $\Delta G^{\ddagger} \mathrm{TS} 3$ & $\Delta G^{\ddagger} \mathrm{TS4}$ & $\Delta G_{\mathrm{TS}(5 \rightarrow 6 \mathrm{a})}$ \\
\hline Gas & 29.6 & 41.9 & 02.6 & 09.3 & 24.2 \\
$\mathrm{CH}_{2} \mathrm{Cl}_{2}$ & 19.9 & 30.1 & 11.6 & 13.0 & 25.6 \\
$\mathrm{CH}_{3} \mathrm{CN}$ & 18.8 & 27.4 & 12.7 & 12.2 & 25.6 \\
THF & 20.1 & 30.5 & 12.0 & 12.7 & 26.4 \\
DMSO & 18.7 & 27.2 & 12.8 & 11.8 & 25.6
\end{tabular}

Since the aldol step is rate-limiting, no $k_{\mathrm{H}} / k_{\mathrm{D}}$ is expected. At the local level, $k_{\mathrm{H}} / k_{\mathrm{D}}$ for the step $\mathbf{6 b} \rightarrow \mathbf{7}$ was estimated at 1.30, irrespective of the solvent. This finding contrasts, in part, an observation in the literature that the reaction medium (solvent) and nature of the aldehyde exert great influence on the absolute value of $k_{\mathrm{H}} / k_{\mathrm{D}} .{ }^{4}$ For instance, $k_{\mathrm{H}} / k_{\mathrm{D}}$ was measured at $2.6 \pm 0.1(5.2 \pm 0.6)$ and $1.0 \pm 0.1(2.4 \pm 0.1)$ in DMSO and THF, respectively, for the DABCO-catalyzed reaction of benzaldehyde ( $p$-nitrobenzaldehyde) and methyl $\alpha-{ }^{2} \mathrm{H}$ acrylate. Except for the reaction of methyl $\alpha-{ }^{2} \mathrm{H}$ acrylate with benzaldehyde in THF, the measured $k_{\mathrm{H}} / k_{\mathrm{D}}$ is two to four times as large as the computationally estimated value.

Hill and Isaacs have measured the kinetic isotope effect $k_{H} / k_{D}$ as $1.03 \pm 0.1$ for the reaction of $\alpha-{ }^{2} \mathrm{H}$ acrylonitrile with acetaldehyde under catalysis by $\mathrm{DABCO} .{ }^{3 b}$ We have estimated the profile of the $\mathrm{Me}_{3} \mathrm{~N}-$ catalysed reaction of acrylonitrile with acetaldehyde in the gas phase and it is shown in Figure 6 . Since the $\Delta \mathbf{G}^{\ddagger}$ for proton transfer in Hofmann fashion $\mathbf{F} \rightarrow[\mathrm{TS} 4] \rightarrow(\mathbf{G}+\mathbf{B})(6.5 \mathrm{kcal} / \mathrm{mol})$ is significantly small in comparison to the highest energy requiring aldol step $(\mathbf{C}+\mathbf{D}) \rightarrow[$ TS2] $\rightarrow E$, the proton transfer is rapid under the reaction conditions and, hence, no $k_{\mathrm{H}} / k_{\mathrm{D}}$ is expected to be observed. Locally, $k_{\mathrm{H}} / k_{\mathrm{D}}$ is 1.35 for this proton-transfer.

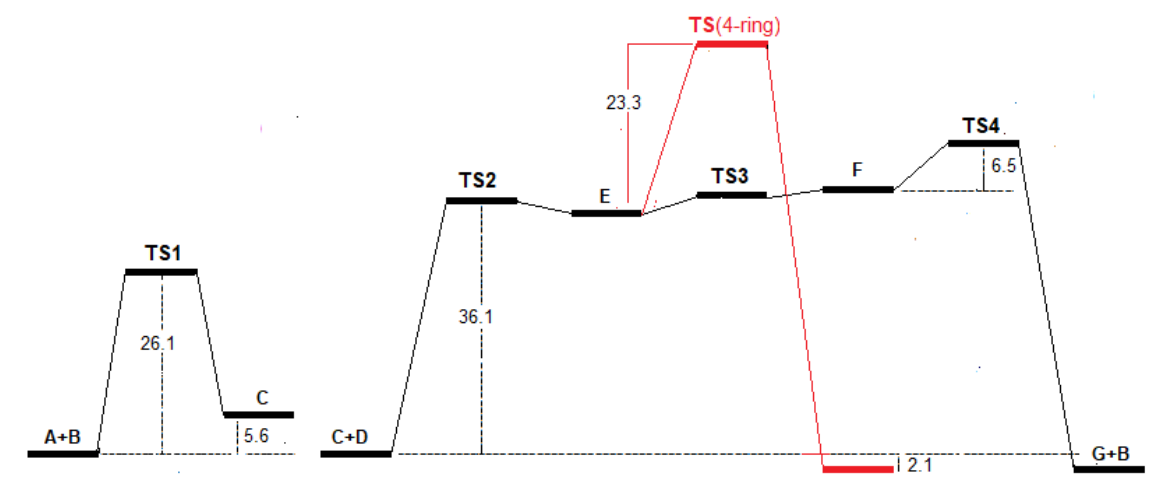

Figure 6. The relative profile of the $\mathrm{M}_{3} \mathrm{~N}$-catalysed $\mathrm{MBH}$ reaction of acrylonitrile with acetaldehyde in gas phase. TS (4-ring) is the TS for the transformation equivalent to $\mathbf{5} \rightarrow \mathbf{6 a}$. $\mathbf{A}=$ acrylonitrile, $\mathbf{B}=\mathrm{Me}_{3} \mathrm{~N}, \mathbf{C}=$ conjugate addition product, $\mathbf{D}=\mathrm{CH}_{3} \mathrm{CHO}, \mathbf{E}=$ aldol product (the alkoxide), $\mathbf{F}=$ ammonium ylide, $\mathbf{G}=\mathrm{MBH}$ product 
It is significant to note that in the above $\mathrm{Me}_{3} \mathrm{~N}$-catalyzed $\mathrm{MBH}$ reaction of acrylonitrile with acetaldehyde, the $\Delta G^{\ddagger}(23.3 \mathrm{kcal} / \mathrm{mol})$ for proton-transfer through four-membered TS structure is $>3.5$ times as high as that for the transfer in Hofmann fashion.

The profile of the $\mathrm{Me}_{3} \mathrm{~N}$-catalyzed $\mathrm{MBH}$ reaction of acrylonitrile with acetaldehyde was also estimated under the solvent effects of DMSO for a comparison. The reaction profile is given in Figure 7 . The $\Delta G^{\ddagger}$ for the conjugate addition and aldol reaction are considerably reduced from those in the gas phase. The $\Delta G^{\ddagger}$ for Hofmann elimination is reduced to under $2.0 \mathrm{kcal} / \mathrm{mol}$, which is insignificant in comparison to 26.4 $\mathrm{kcal} / \mathrm{mol}$ required for proton transfer through four-membered TS structure. The kinetic isotope effect $k_{\mathrm{H}} / k_{\mathrm{D}}$ did not change from that in the gas phase.

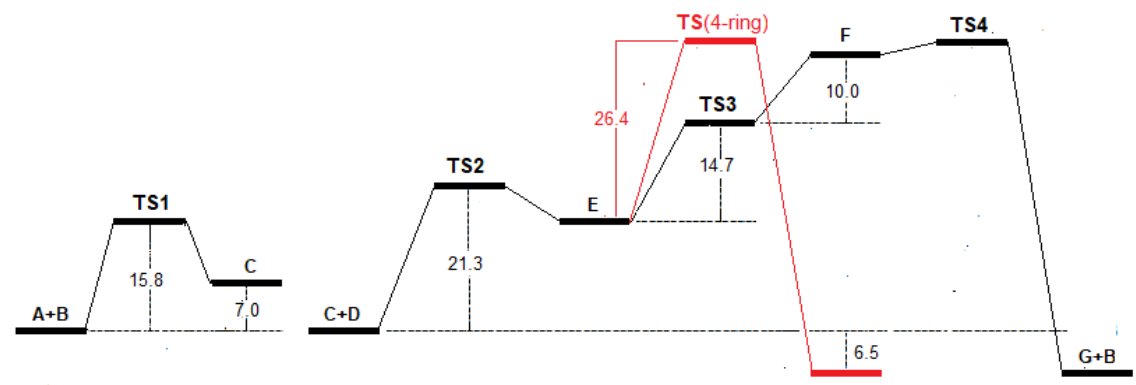

Figure 7. The relative profile of the $\mathrm{M}_{3} \mathrm{~N}$-catalysed $\mathrm{MBH}$ reaction of acrylonitrile with acetaldehyde under the solvent effects of DMSO. $\mathbf{A}=$ acrylonitrile, $\mathbf{B}=\mathrm{Me}_{3} \mathrm{~N}, \mathbf{C}=$ conjugate addition product, $\mathbf{D}=\mathrm{CH}_{3} \mathrm{CHO}, \mathbf{E}=$ aldol product (the alkoxide), $\mathbf{F}=$ ammonium ylide, $\mathbf{G}=\mathrm{MBH}$ product

Having failed at striking $k_{\mathrm{H}} / k_{\mathrm{D}}$ as high as $5.2 \pm 0.6$ reported for the reaction of methyl $\alpha^{-2} \mathrm{H}$ acrylate with $p$ nitrobenzaldehyde under DABCO-catalysis in $\mathrm{DMSO}^{4}$ we wondered whether the catch was in the employment of $p$-nitrobenzaldehyde as the aldol partner or catalysis by DABCO or both. This, coupled with our previous observation that DABCO participated in the Hofmann pathway just as well as $\mathrm{Me}_{3} \mathrm{~N}$ because the bridge-head ylide was formed with great ease, ${ }^{20}$ we ventured to study the reaction of $p$ nitrobenzaldehyde with (a) cyclopentenone under $\mathrm{Me}_{3} \mathrm{~N}$-catalysis and (b) methyl acrylate under DABCOcatalysis, both in the gas phase and under the effects of DMSO as solvent.

The relative profiles of the reaction of $p$-nitrobenzaldehyde with cyclopentenone under $\mathrm{Me}_{3} \mathrm{~N}$-catalysis in the gas phase and under the solvent effects of DMSO are shown in Figures 8 and 9, respectively. Likewise, the relative profiles of the reaction of $p$-nitrobenzaldehyde with methyl acrylate under DABCO-catalysis in the gas phase and under the solvent effects of DMSO are shown in Figures 10 and 11, respectively. The results in regard to $k_{\mathrm{H}} / k_{\mathrm{D}}$ are as follows: 
(a) The $k_{H} / k_{D}$ for Hofmann elimination was estimated at 1.22 in gas phase for the reaction of cyclopentenone with $p$-nitrobenzaldehyde under $\mathrm{Me}_{3} \mathrm{~N}$-catalysis. This number remained unaltered under the solvent effects of DMSO. ${ }^{21}$ Likewise, $k_{H} / k_{D}$ was estimated at 1.36 in the gas phase for proton-transfer through four-membered TS structure, which also remained unaltered under the solvent effects of DMSO.

(b) The $k_{\mathrm{H}} / k_{\mathrm{D}}$ for Hofmann elimination was estimated at 1.34 in gas phase for the reaction of methyl acrylate with $p$-nitrobenzaldehyde under DABCO-catalysis. This number did not change under the solvent effects of DMSO. ${ }^{22}$ Likewise, the $k_{\mathrm{H}} / k_{\mathrm{D}}$ for proton-transfer through four-membered TS structure also did not change from 1.36 for reaction in the gas phase to reaction under the solvent effects of DMSO.

(c) Following the lower energy Hofmann pathway, aldol reaction constitutes to be the ratecontrolling step.

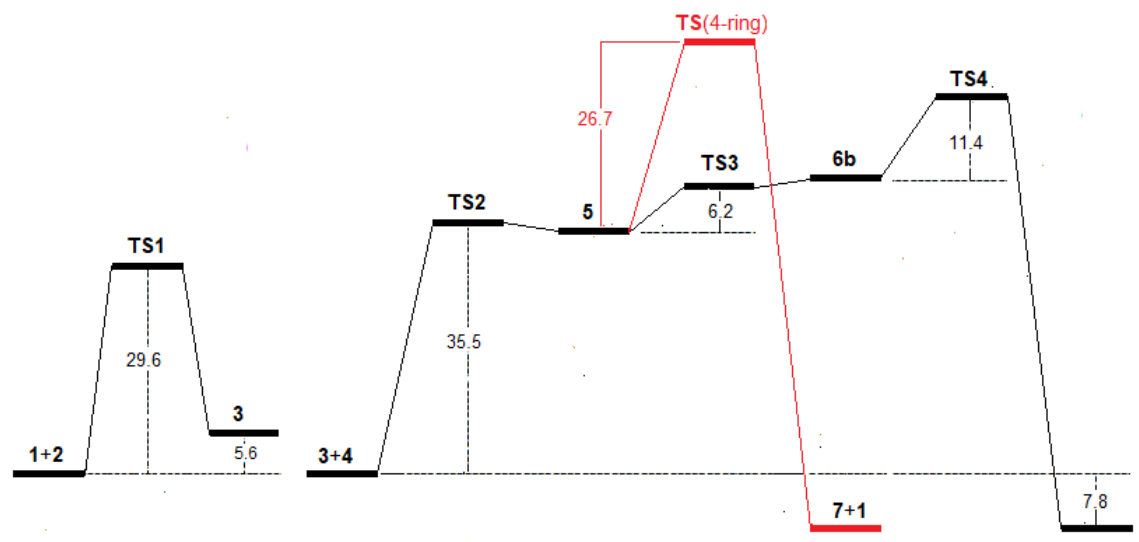

Figure 8. The relative profile of the $\mathrm{M}_{3} \mathrm{~N}$-catalysed $\mathrm{MBH}$ reaction of cyclopentenone with $p$-nitrobenzaldehyde in gas phase. The notations used are the same as those in Figure 1 except 4 which herein represents $p$-nitrobenzaldehyde.

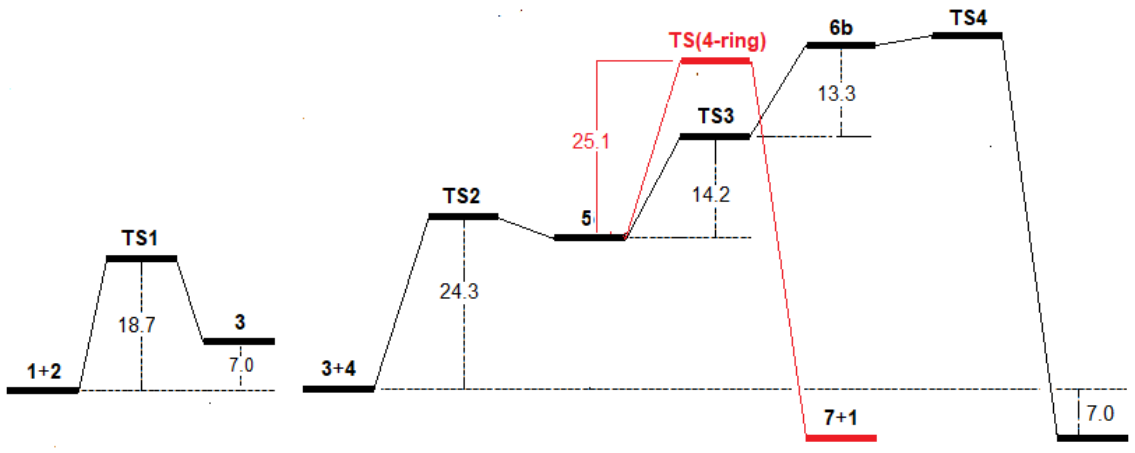

Figure 9. The relative profile of the $\mathrm{M}_{3} \mathrm{~N}$-catalysed $\mathrm{MBH}$ reaction of cyclopentenone with $p$-nitrobenzaldehyde under the solvent effects of DMSO. The notations used are the same as those in Figure 8. 


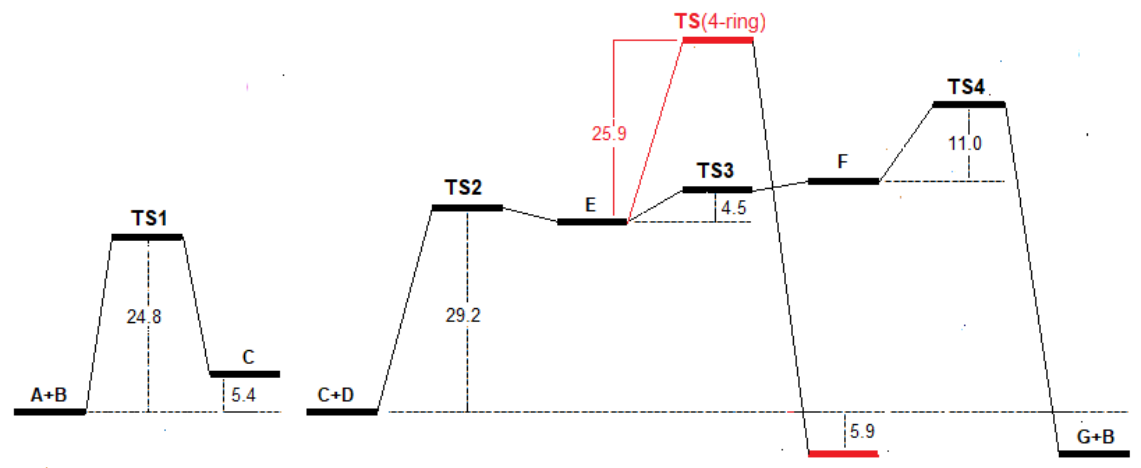

Figure 10. The relative profile of the DABCO-catalysed $\mathrm{MBH}$ reaction of methyl acrylate with $p$-nitrobenzaldehyde in gas phase. $\mathbf{A}=\mathrm{DABCO}, \mathbf{B}=$ methyl acrylate, $\mathbf{C}=$ enolate formed from conjugate addition of $\mathbf{A}$ to $\mathbf{B}, \mathbf{D}=p$-nitrobenzaldehyde, $\mathbf{E}=$ aldol product from reaction of $\mathbf{C}$ with $\mathbf{D}, \mathbf{F}=$ product from proton transfer in $\mathbf{E}$ through seven-membered $\mathrm{TS}, \mathbf{G}=\mathrm{MBH}$ product.

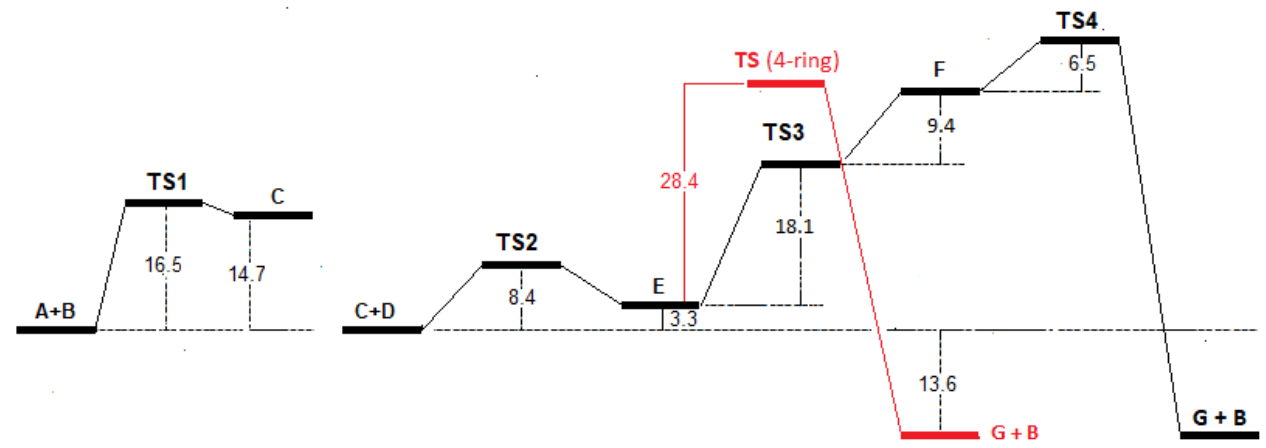

Figure 11. The relative profile of the DABCO-catalysed $\mathrm{MBH}$ reaction of methyl acrylate with $p$-nitrobenzaldehyde under the solvent effects of DMSO. The $\mathbf{A}-\mathbf{G}$ notations are the same as in Figure 10.

(d) From the profile of the DABCO-catalysis reaction of methyl acrylate with $p$-nitrobenzaldehyde under the solvent effects of DMSO given in Figure 11, it is for the first time that the proton-transfer through seven-membered TS structure equivalent to TS3 in Scheme 2 becomes rate-limiting in support of Singleton's findings. ${ }^{2 e}$ The $k_{H} / k_{D}$ estimated for this step, however, is 1.35 (1.37 for proton-transfer through four-membered TS structure).

Thus, DMSO was estimated to affect the rate-controlling step of the reaction of methyl acrylate with $p$ nitrobenzaldehyde under catalysis by $\mathrm{DABCO}$, but the kinetic isotope effect $k_{\mathrm{H}} / k_{\mathrm{D}}$ was nowhere close to what has previously been measured by experiments. The effect of temperature on the course of the reaction is another significant aspect that needs to be looked into.

We return to the mediation of simultaneous proton-transfer to aldol-derived alkoxide and abstraction of proton from $\alpha$-carbon of the activated alkene by hydroxylic solvents through six-membered TS structures proposed by Aggarwal as in Scheme 3. ${ }^{2 \mathrm{~d}}$ TS structures were located for $\mathrm{Me}_{3} \mathrm{~N}$-catalysed reaction of 
cyclopentenone with acetaldehyde and $k_{\mathrm{H}} / \mathrm{k}_{\mathrm{D}}$ estimated at 1.3 for $\mathrm{MeOH}$-catalysis and 1.2 for $\mathrm{H}_{2} \mathrm{O}$ catalysis. In both the instances, the event is barrier-less. A barrier-less event such as this is unlikely to contribute to kinetic isotope effect. Also, for the fact that the aldol event is rate-limiting in the sequence of events, catalysis by hydroxylic solvents by simultaneous proton-transfer and proton-abstraction is not tenable. Any catalysis, if at all, must occur at the aldol level by lowering its activation energy possibly by protonation of the aldehyde. The TS structures for simultaneous proton-transfer to the alkoxide and proton-abstraction from $\alpha$-carbon of the activated alkene by $\mathrm{MeOH}$ and $\mathrm{H}_{2} \mathrm{O}$ are given in the $\mathrm{SI}$ along with geometry coordinates.

We also return to the acetal pathway demonstrated by McQuade and confirmed by its isolation, in some instances, as in Scheme 4. We explored the acetal pathway by studying the DABCO-catalyzed reaction of methyl acrylate with $p$-nitrobenzaldehyde under the solvent effects of DMSO. While McQuade has reported $k_{\mathrm{H}} / k_{\mathrm{D}}=5.2 \pm 0.6$ for this reaction, the number estimated from present investigation is low at 1.35. The TS geometry for abstraction of $\alpha$-proton by the acetal pathway is also given in the SI.

Conclusion. Solvent effects of $\mathrm{CH}_{2} \mathrm{Cl}_{2}, \mathrm{CH}_{3} \mathrm{CN}$, THF and DMSO on the profile of $\mathrm{MBH}$ reaction were estimated and discovered to significantly lower the activation energy of the aldol step which generally is rate-controlling in the sequence of several events. It is only in the instance of DABCO-catalyzed MBH reaction of methyl acrylate with $p$-nitrobenzaldehyde in under the solvent effects of DMSO that the proton-transfer through seven-membered TS structure enroute Hofmann pathway turned rate-limiting with kinetic isotope effect $k_{\mathrm{H}} / k_{\mathrm{D}}$ estimated at 1.35. The energy demand of this proton-transfer was still lower than that through the four-membered TS structure.

The simultaneous proton-transfer to alkoxide and proton-abstraction from $\alpha$-carbon of the activated alkene by hydroxylic solvents such as $\mathrm{MeOH}$ and $\mathrm{H}_{2} \mathrm{O}$ was discovered to be barrier-less. Proton-transfers through seven- and four-membered TS structures are also sufficiently low energy events in comparison to aldol reaction. The proton-transfer, therefore, is not deemed to contribute to the overall kinetics of the reaction, including the kinetic isotope effect. The hydroxylic solvents promote the reaction possibly by better dissolving the polar intermediates to support the follow up reactions or exerting solvent effects to lower activation energy of the rate-controlling aldol step or activating the aldehyde by protonation (even hydrogen bonding) or a mix of some of these. ${ }^{23}$

The acetal route does not contribute to $k_{\mathrm{H}} / k_{\mathrm{D}}$, which has been measured as high as $5.2 \pm 0.6$ by McQuade. ${ }^{4}$ The results of the study of solvent effects and proton-transfers described herein do not support any large influence of the reaction medium (solvent) and the nature of aldehyde on the absolute value of kinetic 
isotope effect as observed previously by others. ${ }^{2 \mathrm{~d}, 4}$ Our results are in resonance with the experimental findings of Hill and Isaacs. ${ }^{3}$ These authors have reported the aldol step as rate-determining step and absolutely no kinetic isotope effect.

\section{ASSOCIATED CONTENT}

\section{Supporting Information}

Supporting Information (SI) available: Cartesian coordinates of the optimized substrates, transition state structures, and Gibbs' free energies.

\section{CONFLICT OF INTEREST}

Author declares no conflict of interest.

\section{ORCID}

Veejendra K. Yadav: 0000-0001-8294-0677

\section{ACKNOWLEDGEMENTS}

The author acknowledges allocation of time on HPC series of supercomputers by the Computer Centre, Indian Institute of Technology Kanpur. This work is dedicated to Professor Deevi Basavaiah for his outstanding contributions to the growth of the $\mathrm{MBH}$ reaction.

\section{REFERENCES}

1. (a) Morita, K.; Suzuki, Z.; Hirose, H. A Tertiary Phosphine-catalyzed Reaction of Acrylic Compounds with Aldehydes. Bull Chem Soc. Jpn. 1968, 41, 2815-2816. (b) Morita, K.; Kobayashi, T. New Addition Reactions of Acrylic Compounds with Fumaric Acid Esters Catalyzed by Tervalent Phosphorus Compounds. Bull Chem Soc Jpn. 1969, 42, 2732-2732. (b) Baylis, A. B.; Hillman, M. E. D. German Patent 1972, 2155113; Chem. Abstr. 1972, 77, 34174q.

2. (a) Basavaiah, D.; Rao, A. J.; Satyanarayana, T. Recent Advances in the Baylis-Hillman Reaction and Applications. Chem. Rev. 2003, 103, 811-891. (b) Aggarwal, V. K.; Fulford, S. Y.; Lloyd-Jones, G. C. Reevaluation of the Mechanism of the Baylis-Hillman Reaction: Implications for Asymmetric Catalysis. Angew. Chem. Int. Ed. 2005, 44, 1706-1708. (c) Buskens, P.; Klankermayer, J.; Leitner, W. Bifunctional Activation and Racemization in the Catalytic Asymmetric Aza-Baylis-Hillman Reaction. J. Am. Chem. Soc. 2005, 127, 16762-16763. (d) Robiette, R.; Aggarwal, V. K.; Harvey, J. N. Mechanism of the Morita-Baylis-Hillman Reaction: A Computational Investigation. J. Am. Chem. 
Soc. 2007, 129, 15513-15525; and references cited therein. (e) Plata, R. E.; Singleton, D. A. A Case Study of the Mechanism of Alcohol-Mediated Morita Baylis-Hillman Reactions. The Importance of Experimental Observations. J. Am. Chem. Soc. 2015, 137, 3811-3826. (f) Pellissier, H. Recent developments in the asymmetric organocatalytic Morita-Baylis-Hillman reaction. Tetrahedron 2017, 73, 2831-2861.

3. (a) Hill, J. S.; Isaacs, N. S. Functionalisation of the $\alpha$ position of acrylate systems by the addition of carbonyl compounds: Highly pressure-dependent reactions. Tetrahedron Lett. 1986, 27, 50075010. (b) Hill, J. S.; Isaacs, N. S. Mechanism of $\alpha$-Substitution Reactions of Acrylic Derivatives. J. Phys. Org. Chem. 1990, 3, 285-288.

4. (a) Price, K. E.; Broadwater, S. J.; Jung, H. M., McQuade, D. T. Baylis-Hillman Mechanism: A New Interpretation in Aprotic Solvents. Org. Lett. 2005, 7, 147-150. (b) Price, K. E.; Broadwater, S. J.; Walker, D. T; McQuade, D. T. A New Interpretation of the Baylis-Hillman Mechanism. J. Org. Chem. 2005, 70, 3980-3987.

5. (a) Yadav, V. K. An alternate energy-conserved pathway for the Morita-Baylis-Hillman (MBH) reaction J. Phys. Org. Chem. 2020 (DOI: 10.1002/poc.4149). (b) A computational study of the Hofmann elimination pathway for the Morita-Baylis-Hillman reaction under DABCO-catalysis. Participation of a bridge-head ylide. Veejendra K. Yadav (2020): ChemRxiv. Preprint. https://doi.org/10.26434/chemrxiv.12117657.v1

6. (a) Cope, A. C.; Mehta, A. S. Mechanism of the Hofmann Elimination Reaction: An Ylide Intermediate in the Pyrolysis of a Highly Branched Quaternary Hydroxide J. Am. Chem. Soc. 1963, 85, 1949-1952. (b) Bartsch, R. A.; Zavada, J. Stereochemical and base species dichotomies in olefin-forming E2 eliminations. Chem. Rev. 1980, 80, 453-494.

7. (a) Denmark, S. E.; Lee, W. Investigations into Transition-State Geometry in the Mukaiyama Directed Aldol Reaction. Chem. Asian J. 2008, 3, 327-341. (b) Brzezinski, L. J.; Rafel, S.; Leahy, J. W. The Asymmetric Baylis-Hillman Reaction. J. Am. Chem. Soc. 1997, 119, 4317-4318.

8. Cantillo, D.; Kappe, C. O. A Unified Mechanistic View on the Morita-Baylis-Hillman Reaction: Computational and Experimental Investigations. J. Org. Chem. 2010, 75, 8615-8626.

9. The $k_{\mathrm{H}} / k_{\mathrm{D}}$ for the proton-transfer step $5 \rightarrow 6$ a was estimated to be 1.36 .

10. Mack, J.; Shumba, M. Rate enhancement of the Morita-Baylis-Hillman reaction through mechanochemistry. Green Chem., 2007, 9, 328-330.

11. Saikia, M.; Sarma, J. C. Baylis-Hillman reaction under solvent-free conditions-Remarkable rate acceleration and yield enhancement Can. J. Chem., 2010, 88, 1271-1276. 
12. R.-Moghadam, K.; Y.-Miri, L. Quick and efficient synthesis of Morita-Baylis-Hillman adducts of isatin derivatives ARKIVOC 2011 (xi) 43-50.

13. Zhao, S.; Zhi, H.; Zhang, M.; Yan, Q.; Fan, J.; Guo, J. Morita-Baylis-Hillman reaction in eutectic solvent under aqueous medium RSC Adv. 2016, 6, 62778-62784.

14. Yu, C.; Liu, B.; Hu, L. Efficient Baylis-Hillman Reaction Using Stoichiometric Base Catalyst and an Aqueous Medium. J. Org. Chem. 2001, 66, 5413-5418.

15. (a) Barrett, A. G. M.; Cook, A. S.; Kamimura, A. Chem. Commun. 1998, 2533-2534. (b) Brzezinski, L. J.; Rafel, S.; Leahy, J. W. Tetrahedron 1997, 53, 16423-16434. (b) Perlmutter, P.; Puniani, E.; Westman, G. Tetrahedron Lett. 1996, 37, 1715-1718. (c) Drewes, S. E.; Emslie, N. D.; Field, J. S.; Khan, A. A.; Ramesar, N. S. Tetrahedron Lett. 1993, 34, 1205-1208.

16. Zhao, Y.; Truhlar, D. G. The M06 suite of density functionals for main group thermochemistry, thermochemical kinetics, noncovalent interactions, excited states, and transition elements: two new functionals and systematic testing of four M06-class functionals and 12 other functionals. Theor. Chem. Acc. 2008, 120, 215-241.

17. (a) Barone, V.; Cossi, M. Quantum Calculation of Molecular Energies and Energy Gradients in Solution by a Conductor Solvent Model. J. Phys. Chem. A 1998, 102, 1995-2001. (b) Cossi, M.; Rega, N.; Scalmani, G.; Barone, V. Energies, structures, and electronic properties of molecules in solution with the C-PCM solvation model. J. Comput. Chem. 2003, 24, 669-681.

18. Gaussian 16, Revision C.01, Frisch, M. J.; Trucks, G. W.; Schlegel, H. B.; Scuseria, G. E.; Robb, M. A.; Cheeseman, J. R.; Scalmani, G.; Barone, V.; Petersson, G. A.; Nakatsuji, H.; Li, X.; Caricato, M.; Marenich, A. V.; Bloino, J.; Janesko, B. G.; Gomperts, R.; Mennucci, B.; Hratchian, H. P.; Ortiz, J. V.; Izmaylov, A. F.; Sonnenberg, J. L.; Williams-Young, D.; Ding, F.; Lipparini, F.; Egidi, F.; Goings, J.; Peng, B.; Petrone, A.; Henderson, T.; Ranasinghe, D.; Zakrzewski, V. G.; Gao, J.; Rega, N.; Zheng, G.; Liang, W.; Hada, M.; Ehara, M.; Toyota, K.; Fukuda, R.; Hasegawa, J.; Ishida, M.; Nakajima, T.; Honda, Y.; Kitao, O.; Nakai, H.; Vreven, T.; Throssell, K.; Montgomery, J. A., Jr.; Peralta, J. E.; Ogliaro, F.; Bearpark, M. J.; Heyd, J. J.; Brothers, E. N.; Kudin, K. N.; Staroverov, V. N.; Keith, T. A.; Kobayashi, R.; Normand, J.; Raghavachari, K.; Rendell, A. P.; Burant, J. C.; Iyengar, S. S.; Tomasi, J.; Cossi, M.; Millam, J. M.; Klene, M.; Adamo, C.; Cammi, R.; Ochterski, J. W.; Martin, R. L.; Morokuma, K.; Farkas, O.; Foresman, J. B.; Fox, D. J. Gaussian, Inc., Wallingford CT, 2016.

19. (a) Reichardt, C. (1990). Solvents and Solvent Effects in Organic Chemistry. Marburg, Germany: Wiley-VCH. pp. 147-181. ISBN 0-89573-684-5 (b) Sundberg, R. J.; Carey, F. A. (2007). Advanced 
Organic Chemistry: Structure and Mechanisms. New York: Springer. pp. 359-376. ISBN 978-0-38744897-8.

20. A computational study of the Hofmann elimination pathway for the Morita-Baylis-Hillman reaction under DABCO-catalysis. Participation of a bridge-head ylide. Veejendra K. Yadav (2020): ChemRxiv. Preprint. https://doi.org/10.26434/chemrxiv.12117657.v1

21. Both in the gas phase and under the solvent effects of DMSO, the kinetic isotope effect $k_{H} / k_{D}$ was estimated at 1.36 for the proton transfer through four-membered TS structure.

22. Both in the gas phase and under the solvent effects of DMSO, the kinetic isotope effect $k_{\mathrm{H}} / k_{\mathrm{D}}$ was estimated at 1.36-1.37 for the proton transfer through four-membered TS structure.

23. Cai, J.; Zhou, Z.; Zhao, G.; Tang, C. Dramatic Rate Acceleration of the Baylis-Hillman Reaction in Homogeneous Medium in the Presence of Water. Org. Lett. 2002, 4, 4723-4725. 


\section{Table of Contents Graphic}

A thorough computational investigation of the amine-catalysed $\mathrm{MBH}$ reaction shows, in conformity with more than three decades old experimental observation, that the reaction must exhibit no kinetic isotope effect $k_{H} / k_{D}$ whether it proceeds through the erstwhile globally popular pathway involving proton transfer through a four-membered TS structure or the recently reported Hofmann pathway. Polar aprotic solvents facilitate the reaction by lowering $\Delta G^{\ddagger}$ of the almost always rate-controlling aldol step. Hydroxylic solvents facilitate the reaction possibly by better dissolving the polar intermediates, not by engaging in simultaneous proton transfer to the aldol-derived alkoxide and abstraction of the $\alpha$-proton of activated alkene.

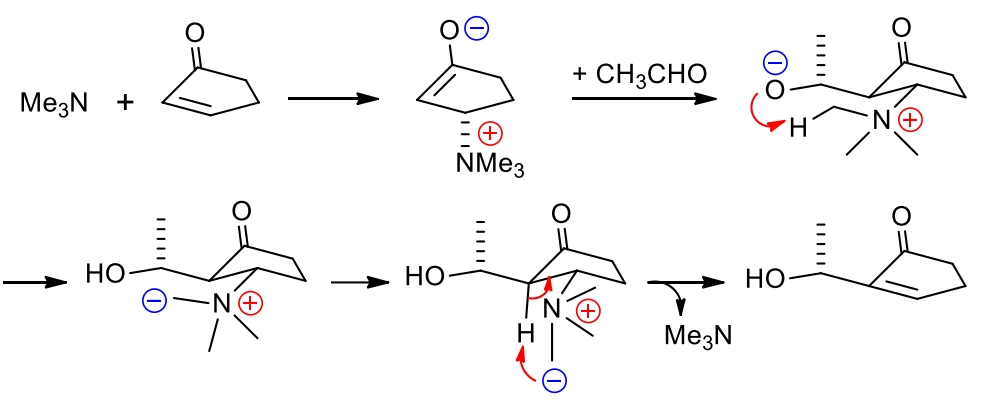

